\title{
Periodo de ventana de virus de hepatitis $B$, detección por biología molecular (NAT)
}

\author{
Window period for hepatitis B, NAT detection
}

\author{
Alejandra Cortés Alegría,* Gabriela Sánchez Díaz* María Rebeca Rivera López,* \\ Jesús Roy Aranda Osorio, Roberto Joaquín Robles Ramírez, Gamaliel Benítez Arvizu*
}

\begin{abstract}
Resumen
Dentro de los agentes infecciosos transmitidos por transfusión en México, cinco son de observancia obligatoria en el tamizaje y detección por los riesgos a la salud y los costos que estos ocasionan: VHC, VHB, sífilis, Chagas y VIH tipo 1 y 2. Dentro de estas ITT (infecciones transmitidas por transfusión) la hepatitis, definida como inflamación de células hepáticas, ocasionada en la mayoría de los casos por virus, es el tema central en el presente trabajo. Se exponen tres casos de infección por VHB en periodo de ventana detectados por medio de la tecnología de ácidos nucleicos; en uno de ellos se logró el seguimiento detallado desde la reactividad a NAT, seroconversión a etapa aguda y diagnóstico final por medio de panel viral. En los tres casos se evidenció la seroconversión mediante panel viral realizado en los laboratorios del Hospital de Especialidades UMAE CMNSXXI. Esto pone en evidencia que la implementación de la tecnología de detección de ácidos nucleicos en los bancos de
\end{abstract}

\begin{abstract}
Among the diseases transmitted by transfusion in Mexico, 5 are mandatory for screening and detection for health risks and the costs they cause: HCV, HBV, syphilis, Chagas and HIV type 1 and 2. Within these TTD (transfusion-transmitted diseases) hepatitis defined as inflammation of liver cells, caused in most cases by viruses is the central issue in this article, three cases detected by technology of nucleic acids are exposed. One of them achieving detailed follow-up from the reactivity to NAT, seroconversion to acute stage and diagnosis by means of viral panel. The other two cases detected in the window period when donating, with subsequent seroconversion evidenced after viral panel control in laboratories of the Specialty Medical Unit of High Specialty Hospital CMNSXXI. Evidence that the implementation of nucleic acid detection technology in blood banks increases transfusion safety, by significantly reducing the residual risk of transfusing blood components found in said window
\end{abstract}

* Banco de Sangre Centro Médico Nacional.

₹ Hospital de Pediatría.

UMAE Centro Médico Nacional Siglo XXI, IMSS. CDMX, México.

Citar como: Cortés AA, Sánchez DG, Rivera LMR, Aranda OJR, Robles RRJ, Benítez AG. Periodo de ventana de virus de hepatitis $B$, detección por biología molecular (NAT). Rev Mex Med Transfus. 2020; 13 (1): 15-21. doi: 10.35366/95495

Rev Mex Med Transfus 2020; 13 (1): 15-21

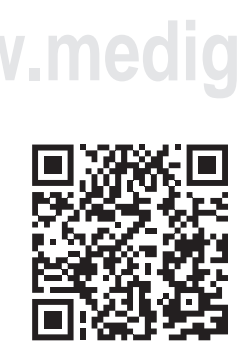

Abreviaturas:

BCSCMNSXXI = Banco Central de Sangre del Centro Médico Nacional Siglo XXI.

NAT = Nucleic acid test (prueba de amplificación de ácidos nucleicos).

$\mathrm{ETT}=$ Enfermedades transmitidas por transfusión.

$\mathrm{VHB}=$ Virus de la hepatitis tipo $\mathrm{B}$.

SS = Seguridad sanguínea.

$\mathrm{RR}=$ Riesgo residual.

www.medigraphic.com/medicinatransfusional 
sangre eleva la seguridad transfusional, disminuye significativamente el riesgo residual de transfundir hemocomponentes que se obtienen en periodo de ventana, evita la consecuente afectación a la salud de los pacientes que pudieron haberse trasfundido con estos hemocomponentes, y permite el seguimiento y la atención oportuna de los donantes al detectarse la infección de manera temprana.

Palabras clave: VHB, NAT, periodo de ventana, ETT, hepatitis $B$, seroconversión, transfusión, tecnología ácidos nucleicos. period, avoiding the consequent impact on the health of people who may have been transfused with these blood components and donors when the infection was detected early.

Keywords: HBV, NAT, window period, TTI, hepatitis $B$, nucleic acid test, seroconversion, transfusion.

\section{Introducción}

La seguridad transfusional puede contemplarse como un engranaje en el que se articulan varios factores principales: la inactivación biológica, la población voluntaria, educada e informada, así como la correcta selección de donantes.' De vital importancia es también el tamizaje y la selección de componentes sanguíneos a través de pruebas de tamizaje.

Se considera que la sangre más segura será la proveniente de donantes voluntarios altruistas de repetición; ${ }^{2}$ en México, la donación de tipo altruista es apenas de $2.3 \%$ mientras que la de reposición familiar alcanza el $97 \%,{ }^{2}$ siendo esta última la que implica mayor riesgo de transmisión de agentes infecciosos por transfusión, 2,3 tales como el virus de la inmunodeficiencia humana, los virus B y C de la hepatitis, Trypanosoma cruzi y Treponema pallidum, las cuales están contempladas en la NOM-253-SSA1-2012 y son de escrutinio obligatorio. ${ }^{4}$

Dentro de los diferentes tipos de hepatitis, la infección por el virus de hepatitis B (VHB) puede ocasionar formas agudas y crónicas de hepatitis. En promedio, el periodo de incubación de la enfermedad es de dos meses, pero puede oscilar desde seis semanas a seis meses, pudiendo cursar asintomática o de manera leve sintomática. ${ }^{5}$ En otras ocasiones se desarrollan síntomas de hepatitis aguda con resolución espontánea (la mayoría de los casos), sin dejar secuelas, en otras puede cursar de forma grave, fulminante o incluso causar la muerte del paciente. En 10\% de los casos la infección es persistente, algunos factores determinantes son la edad al contraerla y la evolución de la enfermedad, la cual con frecuencia es irrelevante en algunos portadores crónicos; sin embargo, en otros casos (10\%) el proceso puede evolucionar, y generar formas graves de enfermedad hepática, como hepatitis crónica severa, cirrosis hepática o incluso cáncer de hígado. ${ }^{5}$

Se estima que actualmente el riesgo residual de transmisión (RRT) definido como la probabilidad (matemática o estadística) de que la transfusión de un componente sanguíneo pueda transmitir un agente infeccioso (viral) después de realizadas las pruebas por métodos de detección directa o indirecta, de antígenos o elementos de la respuesta inmune por transfusión de VHB es: 1 /2,770,000 para USA, a 1/1 millón en el norte Europa, 1/10 millones de donadores en el sur de Europa, y de 1/3,185 donaciones en México. ${ }^{6}$

En la actualidad existen progresos significativos en el tamizaje de las ITT desarrollando pruebas más sensibles y específicas en serología, a la par de la automatización de métodos moleculares como la tecnología NAT (Nucleic Acid Testing)., 
Las pruebas serológicas son pruebas que tienen una gran utilidad para detectar posibles infecciones por VIH, VHC, VHB, Treponema pallidum y Trypanosoma cruzi. Analizan mediante la presencia de antígenos (Ag), anticuerpos (Ab) o ambos en las muestras de suero o plasma de los donadores, son reacciones antígeno-anticuerpo in vitro. Para poder determinar la presencia de los agentes infecciosos en una muestra es necesario que exista una respuesta inmune, la cual puede tardar de dos a seis semanas en presentarse dependiendo del virus/parásito que se esté buscando, por lo tanto, existe el riesgo de que si la infección es muy temprana no se pueda detectar con este tipo de pruebas.

Las pruebas NAT son métodos de biología molecular, en particular para bancos de sangre, que detectan segmentos específicos del material genético de los virus de la inmunodeficiencia humana, virus de la hepatitis $B$ y virus de la hepatitis $\mathrm{C}(\mathrm{VIH}, \mathrm{VHB}$ y $\mathrm{VHC})$ en etapas muy tempranas de la infección, ayudando a reducir el periodo de ventana. En esta metodología se amplifica el ADN o ARN del virus mediante una amplificación mediada por transcripción (TMA), la cual consiste en producir una gran cantidad de copias sintéticas del material genético del virus a partir de un segmento específico del virus que pueda existir en las muestras analizadas, utilizando enzimas como la transcripta inversa y la ARN polimerasa y por medio del uso de moléculas quimioluminiscentes marcadas se detecta la presencia o ausencia del material genético viral mediante una reacción luminosa, este ensayo se denomina Procleix Ultrio Elite Assay y el equipo utilizado es el Procleix Panther System By Hologic and Grifols. Para poder saber específicamente a qué virus es reactiva la muestra se utiliza un ensayo adicional con una sonda discriminatoria específica para el material genético de cada virus. La implementación de las pruebas NAT ha ayudado en las últimas décadas a reducir el riesgo de ITT.
México fue el primer país de Latinoamérica en usar este tipo de tecnología para analizar la sangre de los donadores y en el BCSCMNSXXI se implementó a partir del año 2009, añadiendo una mejora significativa en el tamizaje de la sangre, ${ }^{6}$ aplicándose ambas tecnologías al total de unidades (inicial y dos repeticiones; además, para los reactivos se realizan pruebas confirmatorias o en su caso suplementaria). Pese a no ser obligatoria aún la tecnología NAT y aunque está considerada como de alto costo por paquete obtenido, aproximadamente 120 pesos mexicanos (fuente centro de costos IMSS), en el BCSCMNSXXI se realiza rigurosamente al 100\% de las unidades obtenidas en donación, siguiendo altos estándares de calidad.

Ambas pruebas (serología y NAT) se complementan y son capaces de acortar los periodos de detección de la infección comprendidos desde el ingreso del virus hasta su detección conocida como periodo de ventana. ${ }^{7}$ La tecnología NAT (Nucleic Acid Testing) reduce el periodo de ventana de seis semanas a 16.3 días para el caso de hepatitis B, lo cual aumenta la seguridad sanguínea. ${ }^{8}$

Se presenta un estudio descriptivo observacional en la población de donantes que obtuvieron reactividad a algún marcador en el BCSCMNSXXI en el periodo comprendido de enero de 2015 a septiembre de 2019, en la población de donantes provenientes de los diferentes estados de la República Mexicana. Se incluyen donadores de tipo altruista (2.3\%) y de reposición familiar (97\%).

\section{Presentación de casos}

Se documenta en esta ocasión tres periodos de ventana para $\mathrm{VHB}$.

En el primero se logró evidenciar y documentar por marcadores serológicos y moleculares desde el periodo de ventana hasta el seguimiento y seroconversión de éste para hepatitis $B$, a través de la elevación en sus marcadores realizados en cuatro muestras seriadas. 
En los últimos dos se obtuvo la muestra en periodo de resolución de VHB de manera espontánea, por lo que se logró contactar y explicar su patología, pese a resultar asintomáticos para VHB.

\section{Caso 1}

Masculino de 55 años, ocupación servicios de limpieza en edificio administrativo. Acude a donación de reposición familiar a BCSCMNSXXI.

Durante la entrevista médica inicial niega algún factor de riesgo para infecciones transmitidas por transfusión (ITT). Tras valoración clínica y paraclínica se acepta como donador de sangre total. Se procesan las muestras sanguíneas detectándose serología negativa y NAT reactivo (Tab/a 7).

Se realiza repetición de las pruebas (inicial y dos repeticiones, corroborando reactividad a NAT y no reactividad a serología), ejecutándose prueba discriminatoria, la cual resulta reactiva. En las siguientes tablas se muestra la detección en periodo ventana hasta su seroconversión diagnóstica a hepatitis $B$.

A la obtención de los resultados en BCSCMNSXXI se descarta la unidad dando baja definitiva $y$ destino final, no sin antes tomar las muestras correspondientes para ejecución de las pruebas en tubo y bolsa, respectivamente.

El donante fue citado para realizar segunda entrevista, aplicando interrogatorio médico dirigido intencionado, y con consentimiento informado para ello.

Se encontraron factores de riesgo probables: dos parejas sexuales en un año, una de ellas oca- sional y considerada como de riesgo en los últimos 45 días. Mordedura por humano, la cual causó hemorragia en el cuero cabelludo; remitiendo con cuidados médicos 30 días predonación.

Se realizaron estudios de serología y de biología molecular en tres ocasiones más con un intervalo de nueve días de diferencia entre cada una, documentamos inicialmente periodo de ventana con NAT reactivo y serología negativa.

Se considera como periodo ventana, por lo que se dio seguimiento del caso por medio de panel viral hasta que presenta reactividad serológica concluyendo hepatitis B como etiología (Tabla 2). En las muestras subsecuentes seroconversión y reactividad gradual a AgS VHB, con NAT en persistencia positivo ya con diagnóstico de hepatitis B por panel viral.

El caso fue turnado al Servicio de Gastroenterología para su manejo y tratamiento integral con el respectivo aviso epidemiológico elaborado.

\section{Casos 2 y 3}

Se trata de dos masculinos de la cuarta década de la vida, independientes, con trabajo no relacionado con la salud. Uno administrativo y otro trabajador de campo (seguridad). En las entrevistas se identifica en ambos como único factor de riesgo: homosexualidad. No relacionados entre sí; donaron en diferente mes del mismo año (2018), se muestra cómo cada uno presenta datos de periodo ventana a la donación y cuando fueron entrevistados se aprecian ya en fase de resolución de la hepatitis (Tabla 3), la cual para ambos cursó

Tabla 1: Resultados iniciales en donación discriminatoria.

\begin{tabular}{llllll}
\hline Estudio & Sífilis & Chagas & VIH & VHC & VHB \\
\hline $\begin{array}{l}\text { Quimioluminiscencia (serología) } \\
\text { Ácidos nucleicos por TMA }\end{array}$ & No reactivo & No reactivo & No reactivo & No reactivo & No reactivo \\
\hline VIH = virus de la inmunodeficiencia humana; VHC $=$ virus de la hepatitis C; VHB = virus de la hepatitis B. & No aplica & No reactivo & No reactivo & Reactivo \\
\hline
\end{tabular}


Tabla 2: Resultados subsecuentes del caso 1.

\begin{tabular}{|c|c|c|c|c|c|c|c|}
\hline \multirow[b]{2}{*}{ Determinación } & \multirow{2}{*}{$\begin{array}{c}\text { Donación } \\
\text { Resultado }\end{array}$} & \multicolumn{2}{|c|}{ Muestra 2} & \multicolumn{2}{|c|}{ Muestra 3} & \multicolumn{2}{|c|}{ Muestra 4} \\
\hline & & Resultados & Unidades & Resultados & Unidades & Resultados & Unidades \\
\hline AgHBs & 0.33 & Reactivo & 1.28 & Reactivo & 4.81 & Reactivo & 34.31 \\
\hline VIH & 0.07 & No reactivo & 0.11 & No reactivo & 0.09 & No reactivo & 0.16 \\
\hline $\mathrm{VHC}$ & 0.08 & No reactivo & 0.09 & No reactivo & 0.07 & No reactivo & 0.09 \\
\hline AcTreponema pallidum & 0.06 & No reactivo & 0.05 & No reactivo & 0.05 & No reactivo & 0.05 \\
\hline AcTrypanosoma cruzi & 0.02 & No reactivo & 0.02 & No reactivo & 0.02 & No reactivo & 0.02 \\
\hline \multicolumn{8}{|c|}{ Procleix Ultrio Elite NAT } \\
\hline VIH NAT & 13.76 & Reactivo & 12.81 & Reactivo & 13.65 & Reactivo & 16.20 \\
\hline VHC NAT & 13.76 & Reactivo & 12.81 & Reactivo & 13.65 & Reactivo & 16.20 \\
\hline VHB NAT & 13.76 & Reactivo & 12.81 & Reactivo & 13.65 & Reactivo & 16.20 \\
\hline \multicolumn{8}{|c|}{ Discriminatorias } \\
\hline VHB DISC & 25.77 & Positivo & 22.51 & Positivo & 21.54 & Positivo & 23.49 \\
\hline VIH DISC & 0.16 & Negativo & 0.10 & Negativo & 0.14 & Negativo & 0.25 \\
\hline VHC DISC & 0.1 & Negativo & 0.00 & Negativo & 0.22 & Negativo & 0.10 \\
\hline \multicolumn{8}{|c|}{$\begin{array}{l}\text { Resultados serológicos } \\
\text { Laboratorio especialidades pediatría }\end{array}$} \\
\hline $\mathrm{AgHBs}$ & & Zona gris & & Reactivo & & Reactivo & \\
\hline Ac AntiCORE VHB & & No reactivo & & No reactivo & & No reactivo & \\
\hline Hepatitis C diferencial & & No reactivo & & No reactivo & & No reactivo & \\
\hline Ac Antihepatitis A IgM & & No reactivo & & No reactivo & & No reactivo & \\
\hline Ac Anti-S VHB & & No reactivo & & No reactivo & & No reactivo & \\
\hline Ag E VHB & & No reactivo & & No reactivo & & No reactivo & \\
\hline Ac Anti-E VHB & & No reactivo & & No reactivo & & No reactivo & \\
\hline
\end{tabular}

subclínica. Se decidió dar seguimiento epidemiológico y se canalizaron al área de especialidades para su seguimiento y monitoreo.

Los tres donantes fueron atendidos por personal médico, psicológico y sanitarista con el objetivo de explicar la limitación en futuras donaciones por la reactividad que les fue detectada. También se aclararon dudas al respecto de la infección por $\mathrm{VHB}$, se manejó asignación a especialidades de gastroenterología y epidemiología (población derechohabiente) para monitoreo y seguimiento del caso.

Puntualizando que durante este proceso desde que cualquier prueba de tamizaje resulta en zona gris o reactiva, los hemocomponentes no se liberan y quedan en resguardo para seroteca o para destino final. Gracias a ello se robustecen los mecanismos de prevención para evitar que se transfundan unidades reactivas mediante los candados que el sistema informático del IMSS tiene implementados.

\section{Discusión}

Es la segunda ocasión que se documentan casos en el BCSCMNSXII donde la clínica no muestra datos de infección activa y que se logra 
evitar la transfusión de sangre con reactividad, evitando así los costos y las consecuencias a la salud para los receptores sanguíneos, los cuales de haberse fraccionado y usado podrían haber afectado al menos a tres receptores por cada unidad obtenida.

Los resultados obtenidos son significativos y respaldan la necesidad de usar técnicas moleculares suplementarias para disminuir el riesgo residual en el tamizaje.

Se confirma así el aumento en la seguridad sanguínea a través del empleo de las tecnologías de biología molecular empleadas actualmente (reduciendo el riesgo residual hasta en 83\% empleando pruebas NAT individuales). ${ }^{9}$

Se ofreció atención médica oportuna en etapas iniciales a una enfermedad que quizá no habría sido detectada hasta ya avanzada, obteniendo la oportunidad a través de la concientización en el donante de no diseminar más la enfermedad al conocer su condición médica, aumentando el impacto epidemiológico ${ }^{10}$ que las pruebas moleculares nos brindan.

Extrapolando al aspecto económico se considera que, de acuerdo con el modelo de Noveloy Benítez," se detectaron tres periodos de ventana

Tabla 3: Inicial y segunda muestra de los casos 2 y 3.

\begin{tabular}{|c|c|c|c|c|}
\hline \multirow[b]{2}{*}{ Marcador } & \multicolumn{2}{|c|}{ Caso 2} & \multicolumn{2}{|c|}{ Caso 3} \\
\hline & Donación & Muestra 2 & Donación & Muestra 2 \\
\hline Ag S VHB & 0.47 & $0.180 / N R$ & 0.16 & $0.150 / \mathrm{NR}$ \\
\hline $\mathrm{Ag} E \mathrm{VHB}$ & & Neg & & Neg \\
\hline Ag CORE VHB & & NA & & NA \\
\hline ADN NAT & 17.96 & 0.120 & 15.99 & 0.180 \\
\hline AC anti-S VHB & & 868.27 & & 177.67 \\
\hline Anti-Ag E & & Pos & & Neg \\
\hline Anti-Ag CORE VHB IgM & & No reactivo & & No reactivo \\
\hline Neutralización & \multirow{2}{*}{\multicolumn{2}{|c|}{ Neg }} & \multicolumn{2}{|l|}{ Neg } \\
\hline Discriminatoria & & & \multicolumn{2}{|l|}{25.01} \\
\hline Especialidades CMN SXXI & \multicolumn{2}{|c|}{ VHC NR y VHA 0.37} & \multicolumn{2}{|c|}{ VHC NR y VHA 0.18} \\
\hline
\end{tabular}

Tabla 4: Costos estimados del tamizaje para VHB (en pesos mexicanos) modelo Colombo-Benítez.

\begin{tabular}{|c|c|c|c|c|c|c|c|c|c|}
\hline $\begin{array}{c}\text { Sangres } \\
\text { captadas en } \\
\text { el IMSS } 2015 \\
\text { a } 2019^{*}\end{array}$ & $\begin{array}{l}\text { RR para } \\
\text { VHB en } \\
\text { México** }\end{array}$ & $\begin{array}{l}\text { Sangres } \\
\text { estimadas } \\
\text { con base } \\
\text { en RR }\end{array}$ & Pruebas & $\begin{array}{c}\text { Eficacia } \\
(\%)\end{array}$ & $\begin{array}{l}\text { Probabili- } \\
\text { dad de no } \\
\text { identificar } \\
\text { individuos }\end{array}$ & $\begin{array}{c}\text { Individuos } \\
\text { infectados } \\
\text { por unidad } \\
(3)^{* * *}\end{array}$ & $\begin{array}{c}\text { Costo trata- } \\
\text { miento VHB } \\
\text { anual }\end{array}$ & $\begin{array}{c}\text { Costo anual } \\
\text { de la infec- } \\
\text { ción por } \\
\text { transfusión }\end{array}$ & $\begin{array}{c}\text { Costo } \\
\text { acumulado } \\
\text { a } 10 \text { años }\end{array}$ \\
\hline \multirow[t]{3}{*}{$7,012,205$} & $1: 3,185$ & 113 & NAT + serología & 99.78 & 0 en 100 & 0 & 41,778 & 0 & 0 \\
\hline & & & $\begin{array}{c}\text { Serología } \\
\text { combo }\end{array}$ & 99.05 & 1 en 100 & 3 & 41,778 & 125,334 & $1,253,340$ \\
\hline & & & Serología & 98.00 & 2 en 100 & 6 & 41,778 & 250,668 & $2,506,680$ \\
\hline
\end{tabular}


con NAT que, de no haberse aplicado, se hubieran administrado los componentes obtenidosy transmitido la infección a nueve receptores (tres por unidad trasfundida), lo que implicaría un gasto a 10 años de aproximadamente 1,253,340 pesos por individuo, siendo el gasto en nueve posibles infectados de casi 4 millones de pesos (Tabla 4).

\section{Conclusión}

La seguridad sanguínea, piedra angular en la medicina transfusional, se ve reforzada ampliamente con el uso de técnicas de laboratorio complementarias, actualmente en uso en el BCSCMNSXXI. Mucho se ha hablado de los costos que implica la utilización de NAT como prueba de escrutinio distinta y complementaria a las tecnologías de serología de observancia obligatoria. Sin embargo, al ofrecer acortar el tiempo de periodo de ventana de 40 días usando sólo detecciones de antígenos por serología, a 16 días usando la tecnología de amplificación de ácidos nucleicos, queda justificada e incluso rebasada por el aumento en la seguridad sanguínea dada a nuestras unidades obtenidas. Así como el ahorro en los sistemas de salud al disminuir la incidencia de transmisión por transfusión de estas enfermedades.

\section{Referencias}

1. Bihl F, Castelli D, Marincola F, Dodd RY, Brander C. Transfusiontransmitted infections. J Transl Med. 2007; 5: 25.

2. Rojo MJ. Enfermedades infecciosas transmitidas por transfusión. Panorama internacional y en México. Gac Med Mex. 2014; 150 (1): 78-83.
3. World Health Organization. Universal access to safe blood transfusion [Internet]. 2008. Disponible en: https://www.who.int/ bloodsafety/publications/UniversalAccesstoSafeBT.pdf?ua=1

4. Norma Oficial Mexicana NOM-SSA1-253-2012 "Para la disposición de sangre humana y sus componentes con fines terapéuticos" [Internet]. 2012. Disponible en: http://www.cnts.salud.gob.mx/ descargas/NOM-253-SSAT-2012.pdf

5. Panduro A, Escobedo-Meléndez G, Fierro NA, Ruiz Madrigal B, Zepeda-Carrillo EA, Román S. Epidemiología de las hepatitis virales en México. Salud Pública Méx. 2011; 53: S37-S45. Disponible en: http://saludpublica.mx/index.php/spm/article/view/5023/10010

6. Benítez Arvizu G. Detección de un periodo de ventana de hepatitis B. Periodo de ventana en la medicina transfusional, importancia de la tecnología NAT para la seguridad transfusional. Simposio organizado por Grifols en el Congreso de la Asociación Mexicana de Medicina Transfusional. 21 de septiembre de 2017. pp. 22-27.

7. Villanueva MM. Experiencia de la prueba de NAT en el banco de sangre del Instituto Nacional de Cancerología, México, D.F.. Rev Mex Med Transfus. 2009; 2 (Suppl: 1): 69-71.

8. Camacho-Rodríguez B, NAT en Banco de Sangre ¿Una necesidad o un sobrecosto? IDCBIS, biotecnología e innovación en Salud Bogotá C [internet] 2018. pp. 2-66. Disponible en: https://www. ins.gov.co/Direcciones/RedesSaludPublica/DonacionSangre/ Publicaciones/NAT\%20en\%20Banco\%20de\%20Sangre\%20 costo\%200\%20sobrecosto\%20Dr\%20B\%20Camacho.pdf

9. Vermeulen M, van Drimmelen H, Coleman C, Mitchel J, Reddy R, Lelie N. A mathematical approach to estimate the efficacy of individual-donation and minipool nucleic acid amplification test options in preventing transmission risk by window period and occult hepatitis B virus infections. Transfusion. 2014; 54 (10): 2496-2504.

10. Sánchez Frenes P, Sánchez Bouza MJ, Hernández Malpi S Las enfermedades infecciosas y la transfusión de sangre. Rev Latinoamer Patol Clin. 2012; 59 (4): 186-193.

11. Novelo GB, Benítez AC. Reflexiones sobre la seguridad sanguínea en México. Rev Mex Med Transfus. 2014; 7 (1): 45-50.

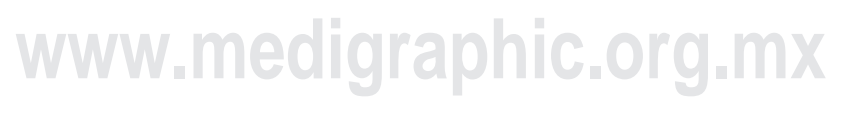

